\title{
INFECÇÕES SEXUALMENTE TRANSMISSÍVEIS EM HOMENS QUE TÊM SEXO COM HOMENS
}

\author{
Rui Campos?', Natividade Rocha², Armando Baptista ${ }^{3}$ \\ 'Interno de Medicina Geral e Familiar/Resident, General and Familiar Medicine, Unidade Saúde Familiar, Vila Nova \\ de Gaia \\ ${ }^{2}$ Assistente Hospitalar de Dermatologia e Venereologia/Consultant, Dermatology and Venereology, Centro Hospitalar \\ Vila Nova de Gaia-Espinho EPE, Vila Nova de Gaia \\ ${ }^{3}$ Chefe de Serviço, Director do Serviço de Dermatologia e Venereologia/Consultant Chief, Head of Department of \\ Dermatology and Venereology, Centro Hospitalar Vila Nova de Gaia-Espinho EPE, Vila Nova de Gaia, Portugal
}

RESUMO - Os homens que têm sexo com homens constituem uma população diversificada, definida pelo sexo e pelo tipo de práticas sexuais, englobando homens com diferentes orientações sexuais.

Os autores efectuaram a revisão dos aspectos epidemiológicos, etiopatogénicos, clínicos, diagnósticos e terapêuticos das infecções sexualmente transmissíveis mais prevalentes nesta população onde se incluem vírus da imunodeficiência humana, sífilis, gonorreia, linfogranuloma venéreo, infecções sexualmente transmissíveis entéricas, papiloma vírus humano, herpes vírus humanos ou hepatite $B$.

A colheita da história sexual é importante na identificação desta população e consequentemente a avaliação da presença de factores de risco para a aquisição de infecções sexualmente transmissíveis e a instituição de medidas preventivas são fundamentais na sua abordagem, de modo a melhorar a saúde entre os homens que têm sexo com homens. Os tratamentos são diversificados conforme a síndrome clínica apresentado e o agente causador, tendo as linhas de orientação mais recentes sido alvo de alterações nos tratamentos dessas patologias.

O reconhecimento desta população e das suas especificidades poderá melhorar o seu acesso aos cuidados de saúde e prevenir os avanços epidemiológicos de algumas das doenças abordadas.

PALAVRAS-CHAVE - Homossexualidade masculina; Doenças sexualmente transmissíveis.

\section{SEXUALLY TRANSMITTED DISEASES IN MEN WHO HAVE SEX WITH MEN}

ABSTRACT - Men who have sex with men are a diverse population, defined by gender and type of sexual practices, encompassing men with different sexual orientations.

The authors conducted a review of epidemiologic, etiopathogenic, clinical, diagnostic and therapeutic aspects of the most prevalent sexually transmitted diseases in this population which include human immunodeficiency virus, syphilis, gonorrhea, lymphogranuloma venereum, sexually transmitted enteric infections, human papilloma virus, herpes virus or human hepatitis $B$.

Improvements in health of men who have sex with men may be accomplished by gathering a complete sexual history in order to identify this population or in the evaluation of risk factors for acquisition of sexually transmitted infections. Recently published guidelines made changes in medical treatments, which vary according to the clinical syndrome presented and it's causative agent.

The recognition of this population and their specificities can improve their access to health care and prevent epidemiological spreads of some of the diseases lectured.

KEY-WORDS - Homosexuality, male; Sexually transmitted diseases. 


\title{
GEIDST
}

\author{
Conflitos de interesse: Os autores declaram não possuir conflitos de interesse. \\ No conflicts of interest. \\ Suporte financeiro: $O$ presente trabalho não foi suportado por nenhum subsídio ou bolsa. \\ No sponsorship or scholarship granted. \\ Direito à privacidade e consentimento escrito / Privacy policy and informed consent: Os autores declaram \\ que pediram consentimento ao doente para usar as imagens no artigo. The authors declare that the patient gave \\ written informed consent for the use of its photos in this article.
}

Recebido/Received - Janeiro/January 2014; Aceite/Accepted - Fevereiro/February 2014

Por decisão dos autores, este artigo não foi redigido de acordo com os termos do novo Acordo Ortográfico.

Correspondência:

Dr. Rui Campos

Rua da Boa Nova 325

4405 Vila Nova de Gaia, Portugal

\section{INTRODUÇÃO}

No fim da década de 80 do século XX o termo homens que têm sexo com homens (HSH) começou a ser utilizado para descrever a realidade de que homens tinham relações sexuais entre si sem qualquer associação de cariz homossexual, sendo actualmente um termo disseminado na literatura médica'.

Assim, os HSH constituem uma população diversificada definida pelo género e tipo de práticas sexuais onde se incluem homens cuja orientação sexual varia entre a homossexualidade, bissexualidade ou heterossexualida$\mathrm{de}^{2}$. É necessário recorrer ao modelo holístico de modo a abordar o comportamento sexual e os relacionamentos desta população cuja evidência mostra que quando comparados com outros homens, os HSH são mais susceptíveis de apresentar infecções de transmissão sexual (IST) incluindo o vírus da imunodeficiência humana $(\mathrm{VIH})$, sífilis, gonorreia, linfogranuloma venéreo, IST entéricas, papiloma vírus humano (HPV), herpes vírus humanos (HSV), hepatite $B$ e possivelmente hepatite $A$ e $C^{3}$.

Desde 2004, após a revisão das linhas de orientação da Organização Mundial de Saúde em relação às IST que estas têm aumentado nos $\mathrm{HSH}$, algumas das razões apontadas foram a diminuição do receio em relação à transmissão do $\mathrm{VIH}$, o uso da Internet como meio eficiente para encontrar parceiros sexuais, o aumento do uso de medicação para a disfunção eréctil e o papel expansivo do sexo oral na transmissão das IST ${ }^{4}$.

Assim o objectivo deste trabalho é rever algumas das principais infecções de transmissão sexual mais comuns nos homens que têm sexo com homens.
Realizou-se uma pesquisa bibliográfica entre Maio e Julho de 2013 recorrendo aos descritores verbais "men who have sex with men" e "Sexually Transmitted Diseases " nas bases de dados de medicina baseada na evidência. Foram também pesquisadas as revistas e livros na área da Dermatologia e Venereologia acerca do tema "Infecções de transmissão sexual".

Na Tabela 1 estão discriminados os principais agentes patogénicos de transmissão sexual e a respectiva síndrome associado nos $\mathrm{HSH}$.

\section{NEISSERIA GONORRHOEAE}

Relativamente à infecção por N. gonorrhea devemo-nos lembrar que é frequentemente causadora de faringites, uretrites e proctites. Os poucos estudos existentes mostraram uma prevalência $15,8 \%$ de testes positivos para $N$. gonorrhea em mais do que um local anatómico pesquisado (uretral, faringe e rectal), sendo mais frequentemente positivo ao nível da uretra $(12,6 \%)$, recto $(6,3 \%)$ e faringe $(1,7 \%)^{5}$.

As infecções genitais são geralmente sintomáticas nos homens, mas quando se refere aos $\mathrm{HSH}$, as infecções extra genitais (faringe e recto) ocorrem mais comummente e são geralmente assintomáticas. A uretrite é classicamente caracterizada pela exsudado mucopurulento e/ou disúria. No caso da proctite, que ocorre nos indivíduos passivos, os sintomas variam do exsudado mucopurulento, tenesmo, obstipação e dor anorrectal. De referir que estes indivíduos apresentam risco superior de adquirir infecção pelo $\mathrm{VIH}$. A faringite 
Tabela 1 Principais síndromes e agentes causadores de infecções sexualmente transmissíveis nos homens que têm sexo com homens².

\begin{tabular}{|c|c|}
\hline AGENTE PATOGÉNICO & SÍNDROMA / DOENÇA \\
\hline \multicolumn{2}{|l|}{ Bacteriano } \\
\hline Neisseria gonorrhoeae & Faringite, proctite, uretrite, epididimite, conjuntivite, gonococcemia \\
\hline Chlamydia trachomatis serovars $D-K$ & Faringite, proctite, uretrite, epididimite, síndrome Reiter \\
\hline Haemophilus ducreyi & Cancróide \\
\hline Chlamydia trachomatis serovars $L 1, L 2, L 3$ & Linfogranuloma venéreo, proctocolite, uretrite \\
\hline Treponema pallidum & Sífilis \\
\hline Calymmatobacterium granulomatis & Donovaniose, granuloma inguinal \\
\hline Campylobacter spp. & Enterite, proctocolite \\
\hline Shigella spp. & Enterite, proctocolite \\
\hline Escherichia coli & Enterite, proctocolite \\
\hline \multicolumn{2}{|l|}{ Vírico } \\
\hline Herpes simplex 1 e 2 & Infecção herpética inicial e recidivante (genital, orolabial e proctite) \\
\hline Papillomavirus humano & $\begin{array}{l}\text { Condiloma acuminado, papiloma laríngeo, displasia anal e } \\
\text { carcinoma anal }\end{array}$ \\
\hline Hepatite A, B e C & Hepatopatia aguda e crónica \\
\hline Vírus Imunodeficiência Humana tipo 1, 2 e subtipo 0 & Infecção VIH crónica, SIDA \\
\hline Mollusco contagiosum & Molusco genital \\
\hline Citomegalovirus & Mononucleose; Proctocolite \\
\hline \multicolumn{2}{|l|}{ Fungo } \\
\hline Candida albicans & Balanite \\
\hline \multicolumn{2}{|l|}{ Ectoparasita } \\
\hline Phthirus pubis & Piolhos \\
\hline Sarcoptes scabei & Escabiose \\
\hline \multicolumn{2}{|l|}{ Protozoário } \\
\hline Cryptosporidium parvum & Enterite, proctocolite \\
\hline Entamoeba histolytica & Enterite, proctocolite \\
\hline Giardia lamblia & Enterite, proctocolite \\
\hline Trichomonas vaginalis & Uretrite \\
\hline
\end{tabular}

gonocócica é normalmente assintomática, podendo por vezes cursar com exsudado ou Linfadenite cervical ${ }^{6}$.

O diagnóstico é laboratorial através do meio de cultura que é usado mais vezes nas infecções da faringe e do recto, enquanto as técnicas de amplificação dos ácidos nucleicos (AAN) que sendo mais sensíveis e menos invasivas, são usadas no diagnóstico das infecções

uretrais. É feita a colheita do primeiro jacto de urina matinal podendo ser recolhido no domicílio, proporcionando maior conforto e adesão por parte do doente ${ }^{2,6}$.

Recentemente foram revistos os tratamentos para a infecção por N. gonorrhea dado o aumento de resistências a alguns antibióticos como as fluoroquinolonas e as cefalosporinas orais (Tabela 2$)^{7}$. 


\section{GEIDST}

Tabela 2 - Tratamento recomendado para infecções pela N. gonorrhea.

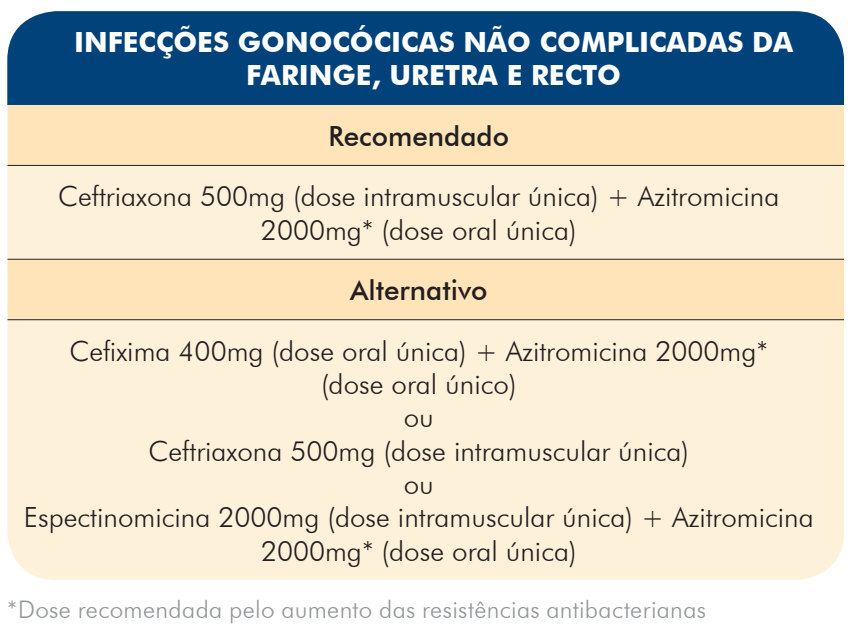

Para todos os doentes com gonorreia devem ser feitos esforços de modo a tratar todos os parceiros sexuais que tiveram contacto nos sessenta dias prévios ${ }^{8}$. Sendo também recomendada a repetição dos testes diagnósticos para excluir reinfecção, sendo realizados duas semanas após ter completado o tratamento ${ }^{2,7}$.

\section{CHLAMYDIA TRACHOMATIS}

Semelhante à N. gonorrhea, a Chlamydia trachomatis é responsável por infecções da faringe, da uretra e do recto?

Epidemiologicamente, é o agente bacteriano mais frequentemente responsável pelas IST nos países desenvolvidos, sendo a maioria, clinicamente assintomática. Existem diversos serotipos que estão associados ao tracoma (serotipos $A, B, B a$ e $C$ ), a síndromes génito-urinários e rectais (serotipos $\mathrm{D}$ a K) e o linfogranuloma venéreo (L1, L2a, L2b E L3) $)^{7,9}$.

No homem, é o principal responsável pelas uretrites não-gonocócicas e a complicação mais comum é a epididimite em indivíduos jovens. A sintomatologia, quando presente, surge após 7 a 21 dias de incubação, com disúria e exsudado uretral seroso e esbranquiçado (conhecido como "leitoso matinal") .

Outra manifestação clínica é a proctite clamidial, definida como uma inflamação da mucosa rectal distal, incomum e quase exclusivamente associada aos $\mathrm{HSH}$ passivos ${ }^{10}$ e que pode surgir associada aos serotipos D e K, no entanto é mais ligeira quando comparada com a infecção pelos serotipos $L$, responsáveis pelo linfogranuloma venéreo (LGV) ${ }^{9}$. Esta consiste numa IST
Tabela 3 - Tratamento das infecções por C. trachomatis.

\begin{tabular}{c}
\hline INFECÇÕES POR C. TRACHOMATIS \\
Infecção urogenital \\
\hline Azitromicina $1000 \mathrm{mg}$ (dose oral única) \\
\hline Infecção rectal \\
\hline Doxiciclina 100mg (dose bidiária durante 7 dias) \\
\hline Linfogranuloma venéreo \\
\hline Doxiciclina 100mg (dose bidiária durante 21 dias) \\
ou
\end{tabular}

cujas manifestações clínicas incluem 3 estadios. No primeiro surge uma pápula/úlcera genital transitória, no segundo ocorre disseminação linfática inguinal exuberante, supurativa e muito dolorosa (bubão) e, por fim, no terceiro estadio surge o síndrome anorrectal caracterizado por proctite hemorrágica que poderá mimetizar doença inflamatória intestinal, com abcessos, fistulas perirrectais, estenoses e rectorragias ${ }^{2}$. O não tratamento desta doença poderá, em estádios avançados, levar a fibrose e estreitamento do tracto anogenital ${ }^{11}$.

O diagnóstico é baseado na clínica apresentada associada, se possível, a técnicas de amplificação dos ácidos nucleicos (AAN) capazes de distinguir os diferentes serotipos de C. trachomatis. Para além da AAN existem outros exames disponíveis para o diagnóstico como a imunofluorescência directa (baixa sensibilidade e especificidade) e a culturar ${ }^{12,13}$.

É importante reconhecer o risco de co-infecção com N. Gonorrhea que ocorre nestes doentes estando aconselhado o tratamento empírico de ambos os agentes infecciosos. A Tabela 3 mostra os regimes terapêuticos mais comuns ${ }^{12,13}$.

É fundamental o tratamento dos indivíduos infectados e dos parceiros sexuais dos últimos 3 meses.

\section{SíFILIS}

No capítulo das IST que têm vindo a aumentar a sua incidência, inclui-se a sífilis, causada pelo Treponema pallidum. São variadas as razões para o seu aumento, entre elas os comportamentos sexuais de risco (múltiplos parceiros sexuais), uso de Internet, o consumo de drogas $^{14}$ e particularmente os doentes $\mathrm{VIH}$ e HSH${ }^{15}$. 
Tabela 4 - Estadios da sífilis, evolução e manifestações.

\begin{tabular}{|c|c|c|c|}
\hline ESTÁDIO & EVOLUÇÃO & \multicolumn{2}{|c|}{ MANIFESTAÇÕES } \\
\hline Secundária & 1 a 3 meses & $\begin{array}{l}\text { Exantema maculopapular/papuloescamoso } \\
\text { com atingimento das palmas e plantas, } \\
\text { artralgias, pápulas violáceas perianais } \\
\text { (condiloma lata), fadiga, linfadenopatia } \\
\text { generalizada, cefaleias, mialgias e faringite }\end{array}$ & $\begin{array}{l}\text { Sífilis anular, irite, sífilis pustular, } \\
\text { pirexia, alopecia sifilítica e sífilis } \\
\text { ulcero-nodular }\end{array}$ \\
\hline Latente precoce & $\begin{array}{l}\text { Após estádio primário ou } \\
\text { secundário } \\
1 \text { ano ou menos } \\
\text { assintomático }\end{array}$ & Ausência de sintomas ou sinais clínicos & $\begin{array}{l}\text { Ausência de sintomas ou sinais } \\
\text { clínicos }\end{array}$ \\
\hline Terciária & Meses a anos & $\begin{array}{l}\text { Neurossífilis tardia Sífilis cardiovascular, sífilis } \\
\text { gomatosa }\end{array}$ & \\
\hline
\end{tabular}

A sífilis, conhecida como a grande imitadora, é uma doença sistémica caracterizada pelo aparecimento de uma úlcera indolor, limpa e de consistência cartilaginosa no local da inoculação (genital ou extra-genital), também denominada de cancro duro, que cura espontaneamente até normalmente um mês ${ }^{16}$.

A Tabela 4 resume as características de cada uma das fases da doença.

Nos $\mathrm{HSH}$, a probabilidade de o diagnóstico ser feito em fases mais tardias é superior, pois as lesões podem passar despercebidas na medida em que muita das vezes não são observadas as mucosas oral e/ou anal ${ }^{2}$.

O diagnóstico definitivo de sífilis é feito pela demonstração da existência de espiroquetas com recurso à microscopia de fundo escuro de exsudados de lesões no estádio primário ou secundário ou através de técnicas de AAN de exsudado de úlcera ou de biopsia de lesões. Na maioria dos casos o diagnóstico é feito pela combinação da clínica com os resultados das serologias (diagnóstico de probabilidade) como mostra a Tabela 52,16.

A penicilina intramuscular continua a ser o tratamento de escolha. A sífilis primária, secundária e latente precoce, deverão ser tratadas com penicilina em dose única intramuscular $(2,4$ milhões de unidades internacionais). No caso da sífilis latente tardia ou de duração desconhecida, o tratamento será de 3 doses (dose/semana), num total de 7,2 milhões de unidades

Tabela 5 - Testes diagnósticos utilizados na sífilis.

\begin{tabular}{|c|c|c|c|}
\hline TESTE & EXPLICAÇÃO & VANTAGENS & LIMITAÇÕES \\
\hline $\begin{array}{l}\text { Microscopia de fundo } \\
\text { escuro }\end{array}$ & $\begin{array}{l}\text { Visualização directa das } \\
\text { espiroquetas }\end{array}$ & $\begin{array}{l}\text { Diagnóstico imediato } \\
\text { Notificação precoce dos parceiros }\end{array}$ & $\begin{array}{l}\text { Lesões orais (espiroquetas não } \\
\text { venéreas, comensais na orofaringe) }\end{array}$ \\
\hline $\begin{array}{l}\text { Serologia não } \\
\text { treponémica (VDRL, } \\
\text { RPR) }\end{array}$ & $\begin{array}{l}\text { Detecta anticorpo } \\
\text { anticardiolipina no } \\
\text { sangue }\end{array}$ & $\begin{array}{l}\text { Barato } \\
\text { Títulos correlacionam-se com sucesso/falha do } \\
\text { tratamento } \\
\text { Marcador de actividade da doença }\end{array}$ & $\begin{array}{l}\text { Período janela com falsos negativos } \\
\text { Falsos positivos (idade, doenças auto- } \\
\text { imunes, malária, VIH, herpes genital) }\end{array}$ \\
\hline $\begin{array}{l}\text { Serologia } \\
\text { treponémica (TPHA) }\end{array}$ & $\begin{array}{l}\text { Detecta anticorpos do } \\
\text { T. pallidum no sangue }\end{array}$ & $\begin{array}{l}\text { Confirmatório } \\
\text { Reactivo mais precocemente que testes não } \\
\text { treponémicos }\end{array}$ & $\begin{array}{l}\text { Custo superior aos não treponémicos } \\
\text { Período janela }\end{array}$ \\
\hline
\end{tabular}




\section{GEIDST}

internacionais de penicilina. No caso de alergia, o fármaco alternativo é a doxiciclina (100 miligramas, duas vezes por dia durante 14 dias) ${ }^{13}$.

A avaliação da resposta ao tratamento é feita pela variação nos níveis (título) de VDRL ou RPR (diferença de 2 diluições) pesquisados aos 6 e 12 meses após o tratamento inicial ${ }^{13}$.

Nos HSH activos, está recomendada a realização de serologias para a sífilis anualmente ${ }^{15}$.

\section{INFECÇÕES VÍRICAS}

O desenvolvimento de teste serológicos específicos para a determinação do herpes simplex tipo 1 (VHS-1) e tipo 2 (VHS-2) e o papel destes vírus como facilitadores na transmissão do $\mathrm{VIH}$, renovaram interesse na epidemiologia do herpes nos $\mathrm{HSH}^{2}$.

O VHS tornou-se a principal causa de úlcera genital nos países desenvolvidos, no entanto, por ser uma infecção subclínica em grande parte das vezes, é ainda subestimada ${ }^{17,18}$.

A transmissão é relativamente rápida e pode ocorrer em períodos em que os sintomas não estão presentes ${ }^{18}$. Clinicamente, o quadro clínico varia de prurido recidivante, eritema, dor neuropática genital, a aglomerados vesiculares e ulcerações dolorosas. As lesões localizam-se ao nível do pénis, escroto, área perineal, ânus e recto ${ }^{2,18}$.

O diagnóstico deverá ser confirmado com recurso a testes laboratoriais, pois a apresentação clássica de múltiplas vesículas numa base eritematosa nem sempre está presente. Os testes laboratoriais incluem cultura do vírus (difícil), serologias específicas e testes de AAN das secreções ${ }^{2,18}$.

A história natural da infecção por VHS consiste em três etapas ${ }^{18}$ :

1) Infecção primária ou primo-infecção, em que o individuo é seronegativo para ambos os tipos de HSV e contrai a doença pela primeira vez;

2) Primeiro episodio clínico, que consiste em contrair a infecção pelo HSV tendo imunidade prévia;

3) Doença sintomática recidivante.

O tratamento da infecção por HSV é feito com recurso a agentes antivíricos que apresentam resultados semelhantes entre si em termos de eficácia.

A Tabela 6 mostra os regimes terapêuticos recomendados para a infecção primária e recidivas ${ }^{19}$.

O seguimento, tratamento e aconselhamento dos parceiros também deve ser realizado ${ }^{13}$.

\section{Tabela 6 - Tratamento farmacológico no herpes} genital.

\section{INFECÇÃO PRIMÁRIA DE HERPES GENITAL*}

Aciclovir 400mg (3 tomas diárias durante 5 dias) ou

Aciclovir 200mg (5 tomas diárias durante 5 dias) ou

Valaciclovir 500mg (†oma bidiária durante 5 dias)

\section{RECIDIVA}

Aciclovir 200mg (5 tomas diárias durante 5 dias)

ou

Aciclovir 400mg (3 tomas diárias durante 3 a 5 dias) ou

Aciclovir $800 \mathrm{mg}$ (3 tomas diárias durante 2 dias) ou

Valaciclovir $500 \mathrm{mg}$ (†oma bidiária durante 3 a 5 dias)

TERAPÊUTICA SUPRESSIVA PARA HERPES GENITAL RECIDIVANTE ( $>6$ CRISES ANO)

Aciclovir 400mg (toma bidiária) ou

Valaciclovir 500mg (†oma diária), se $<10$ crises/ano ou

Valaciclovir $750 \mathrm{mg}$ (†oma bidiária), se $>10$ crises/ano ou

Valaciclovir 1000mg (toma diária), se $>10$ crises/ano

*10 dias de tratamento e duplicação das doses se VIH positivo

A principal causa de consulta entre os $\mathrm{HSH}$ são os condilomas acuminados cujo agente responsável é o papilomavírus humano (HPV). Existem dezenas de serotipos responsáveis por verrugas anogenitais, no entanto, os mais frequentemente envolvidos são o subtipo 6 e 11.

O quadro clínico pode variar desde uma infecção assintomática (ou subclínica), ao aparecimento de verrugas perianais, anais internas e ou externas e carcinoma anorrectal (raramente peniano que é mais frequente entre $\mathrm{HSH}, \mathrm{VIH}$ positivo), neste último caso os subtipos mais frequentemente envolvidos são o $16,18,31,33$, serotipos com maior potencial oncogénico ${ }^{2}$.

As verrugas dependendo da localização e tamanho poderão ser dolorosas ou pruriginosas. $O$ seu aspecto macroscópico varia de plana, papulosa ou exofítica ${ }^{13}$.

A exposição ao vírus é, maioritariamente, por contacto sexual, mas não exclusivamente. Estudos de prevalência mostraram que mais de $80 \%$ da população sexualmente activa foi exposta ao HPV, mas apenas uma percentagem desenvolve lesões clínicas².

A infecção anal com HPV tem sido um tópico de interesse crescente, apesar do rastreio não ter sido 
adoptado universalmente. No caso dos $\mathrm{HSH}$ poderá ser importante na medida em que o carcinoma de células escamosas do ânus é duas vezes mais comum do que na restante população ${ }^{20}$. $O$ rastreio poderá ser realizado de forma equivalente ao que é usado no cancro cervical uterino nas mulheres, com recurso ao exame citológico de esfregaço rectal ${ }^{20}$.

Outra variante mais recentemente estudada é o carcinoma de células escamosas da orofaringe que se tem tornado mais comum, associado principalmente ao tipo oncogénico 16, apesar da prevalência estimada ser menor quando comparada com a da região anal $^{21}$.

O seu diagnóstico é clínico, através da inspecção, mas poderá ser confirmado por biopsia particularmente se:

1) O diagnóstico é duvidoso;

2) Não há resposta ao tratamento ou até ocorre agravamento;

3) Verruga com aspecto atípico;

4) Doente imunodeprimido;

5) Verruga com outras características como pigmentação, endurecimento, fixação, ulceração ou sangramento ${ }^{13}$.

O tratamento está indicado nas lesões macroscópicas (por exemplo, verrugas anogenitais) ou em lesões com risco pré-maligno (papulose Bowenóide, caracterizada por pápulas e placas violáceas planas e discretas). As lesões subclínicas podem curar espontaneamente e portanto o uso de antivíricos não é recomendado; também não esta recomendado o tratamento das lesões subclínicas detectadas por outros métodos, como aplicação de ácido acético ou testes de pesquisa de DNA do $\mathrm{HPV}^{13}$. Sem tratamento poderá ocorrer cura espontânea, permanecer inalterada,

Tabela 7 - Tratamento das verrugas externas.

\begin{tabular}{c}
\hline VERRUGAS GENITAIS EXTERNAS \\
Aplicada pelo paciente \\
\hline Imiquimod creme a $5 \%$ \\
\hline Aplicado pelos profissionais de saúde \\
\hline Criocirurgia com azoto líquido \\
(a cada 1 ou 2 semanas de intervalo) \\
ou \\
Remoção cirúrgica \\
ou \\
Acido tricloroacético (80 a $90 \%)$ \\
ou \\
Laser CO2 / electrocirurgia
\end{tabular}

aumentar quer em tamanho mas também em número, ou ficar latente dentro das células epiteliais.

Os regimes terapêuticos existentes são vários (ver Tabela 7) e os factores que influenciam a sua escolha são o tamanho, número, local anatómico, morfologia, preferência do paciente, custo do tratamento, conveniência, efeitos adversos e experiencia do profissional de saúde ${ }^{13,22}$.

A presença de verrugas intra-anais pode ser indicadora de sexo anal receptivo pelo que deverá ser activamente discutido e rastreadas outras IST (gonorreia rectal, clamídia rectal e sífilis) ${ }^{13}$.

A prevenção actual pode ser feita com o recurso a vacinas com os subtipos de HPV de elevado risco oncogénico (16 e 18) e também com os subtipos mais comuns nas verrugas genitais com baixo potencial oncogénico (6 e 11). Recomendar o uso de preservativo de modo a diminuir o risco de transmissão da doença é importante, no entanto, a protecção não é total. $\mathrm{Na}$ presença de verrugas intra-anais, rectorragia ou obstrução rectal, será necessário a referenciação aos cuidados de saúde secundários ${ }^{13}$.

\section{HEPATITES A E B}

As hepatites $A$ e $B$, cujos agentes responsáveis pela transmissão são o vírus hepatite $A(V H A)$ e o vírus da hepatite $B(V H B)$ respectivamente, são IST através da via oral e anal ${ }^{2}$. Estas duas doenças estão incluídas nas IST cuja prevenção pode ser realizada através da vacinação, como também sucede com o HPV ${ }^{13}$.

A hepatite $A$ apresenta um período de incubação de cerca de 28 dias, sendo frequentemente autolimitada. $O$ diagnóstico de hepatite $A$ aguda apenas é confirmado recorrendo ao teste serológico que irá demonstrar anticorpo $\lg M$ positivo para $\circ \mathrm{VHA}$. O tratamento será de suporte para os sintomas que possam surgir, estando a hospitalização salvaguardada para doentes que apresentem desidratação ou insuficiência hepática aguda $^{13}$.

A hepatite $B$, cujo período de incubação é mais extenso, e que vai de 6 semanas aos 6 meses, pode ser uma infecção autolimitada ou tornar-se $\mathrm{crónica}^{13}$. $\mathrm{O}$ estado de portador crónico pode conduzir a processos de hepatite crónica, cirrose hepática e hepatocarcinoma. O diagnóstico é serológico, sendo possível varias combinações e consequentemente interpretações serológicas (Tabela 8).

A prevenção primária é feita com a vacinação, que em Portugal, faz parte do plano nacional de vacinação. 


\section{GEIDST}

Tabela 8 - Interpretação dos marcadores serológicos na hepatite B.

\begin{tabular}{|c|c|c|c|c|}
\hline \multicolumn{4}{|c|}{ MARCADORES SEROLÓGICOS } & \multirow[t]{2}{*}{ INTERPRETAÇÃO } \\
\hline HBsAg & Total anti-HBc & $\lg M$ anti-HBc & Anti-HBs & \\
\hline- & - & - & - & Nunca infectado \\
\hline+ & - & - & - & Infecção aguda precoce; transitório após vacinação \\
\hline+ & + & + & - & Infecção aguda \\
\hline- & + & + & - & Infecção aguda resolvida \\
\hline- & + & - & + & Recuperado de infecção passada e com imunidade \\
\hline+ & + & - & - & Infecção crónica \\
\hline- & + & - & - & Falso positivo; infecção passada; transferência passiva mãe-feto; \\
\hline
\end{tabular}

O tratamento da hepatite B é específico e ultrapassa o objectivo desta revisão.

No caso da hepatite $C$, é uma forma importante de hepatite de transmissão sexual, principalmente em $\mathrm{HSH}$ com VIH.

\section{PARASITOSES E INFECÇÕES ENTÉRICAS}

A Giardia lamblia, a Entamoeba histolytica, a Shigella species e o cryptosporidium parvum são agentes importantes responsáveis por queixas de gastroenterite, dor abdominal, melenas e rectorragias, diarreia e tenesmo nos $\mathrm{HSH}^{2}$. Assim, na presença da sintomatologia previamente descrita dever-se-ão realizar exame microbiológico das fezes (com 3 amostras), para pesquisar bactérias entéricas e parasitas (ovos incluídos). $\mathrm{Na}$ Tabela 9 mostram-se as principais síndromes e agentes causadores.

Em HSH com queixas de proctite e suspeita de se tratar de uma IST, em que não há apoio laboratorial disponível ou no caso de sintomatologia persistente e testes laboratoriais negativos, deve ser tratado empiricamente (tratamento sindrómico) que inclui terapêutica quádrupla com doxiciclina (100 miligramas, duas tomas diárias, per os, durante 7 dias), ceftriaxone (500 miligramas, injecção intramuscular única), valaciclovir (500 miligramas, duas tomas diárias, per os, durante 5 a 10 dias) e penicilina (benzatinica, 2.400 .000 de unidades, injecção intramuscular única).

\section{CONCLUSÃO}

São várias as IST que podem afectar os $\mathrm{HSH}$, neste trabalho apenas se encontram caracterizadas as mais frequentemente encontradas na clínica.

Para que o clinico aborde, diagnostique, trate e previna as IST é importante que obtenha a história sexual do paciente, incluindo orientação sexual e receios ou preconceitos estabelecidos.

O papel do clinico na prevenção é importante ao discutir temáticas relacionadas com a redução do risco e praticas sexuais seguras. Na prevenção primária, a

Tabela 9 - Infecções entéricas.

\section{INFECÇÕES SEXUALMENTE TRANSMISSÍVEIS CAUSADORAS DE:}

\begin{tabular}{l|l|l}
\multicolumn{1}{c|}{ Proctite distal } & \multicolumn{1}{c}{ Proctocolite } & \multicolumn{1}{c}{ Enterites } \\
\hline Neisserea gonorreia & Shigella spp. & Giardia lamblia \\
Clamidia trachomatis (genótipo $D, K$ e L) & Campylobacter & Cryptosporidium spp \\
Treponema pallidum & Salmonella spp. & Micosporidium spp. \\
Herpes simplex virus & Entamoeba histolytica & Hepatite A \\
& Cryptosporidium spp & \\
& Escherichia Coli & \\
& Citomegalovirus &
\end{tabular}


vacinação pode desempenhar um papel importante, nomeadamente ao nível da hepatite $A$ e $B$, meningococcemia ou até $\mathrm{HPV}^{13,23}$.

A consulta deverá garantir a confidencialidade, empatia, atitude não crítica e linguagem acessível.

Nos HSH é obrigatório a pesquisa de manifestações de IST (próprio e parceiros), conhecer a fonte dos parceiros sexuais na comunidade e realizar exames laboratoriais de rotina assegurando a acessibilidade destes doentes aos cuidados de saúde e aplicar as medidas preventivas recomendadas, como sejam a avaliação anual de todos os $\mathrm{HSH}$ sexualmente activos ${ }^{13}$ :

1) Serologia VIH, sífilis, HSV-2 (se desconhecido), estudo imunológico para a hepatite $A$, hepatite $B$ e hepatite $C$ (se história actual ou passada de consumo de drogas intravenosas);

2) Avaliação de infecção uretral (se parceiro insertivo) por N. gonorrhea e C. trachomatis - 1ㅇ jacto de urina da manhã para AAN;

3) Avaliação de infecção rectal (se parceiro receptivo) por N. gonorrhea e C. trachomatis - cultura ou AAN;

4) Avaliação de infecção faríngea por gonococos (se exposição oral) - cultura ou AAN.

A avaliação mais frequente (de 3 a 6 meses) é aconselhada em indivíduos $\mathrm{com}^{13}$ :

a) Múltiplos parceiros sexuais;

b) Com parceiros anónimos;

c) Prática de sexo anal e consumo de drogas;

d) Prática de sexo anal e anfetaminas;

e) Parceiros incluídos em a, b, c ou d.

É importante por tudo isto, realizar uma abordagem integrada da saúde sexual, de modo a melhorar a saúde e prevenir doenças como VIH e IST entre os HSH. Para isto contribuirá a criação de normas de actuação nestes grupos de risco para que os médicos estejam cada vez mais alerta sobre a temática e abordem a saúde sexual dos seus doente de forma a criar uma relação médico-doente saudável $\left.\right|^{3,23}$.

\section{BIBLIOGRAFIA}

1. Altman D, Aggleton P, Williams M, Kong T, Reddy $V$, Harrad $D$ et al. Men who have sex with men: stigma and discrimination. The Lancet, 2012; 380: 439-45.

2. Wong William. Sexually Transmitted Diseases in Men Who Have Sex with Man. In: Klausner JD, Hook EW, editors. Current Diagnosis \& Treatment of Sexually Transmitted Diseases. $1^{\text {st }}$ ed. New York: McGraw-Hill Medical; 2007. p. 167-76.

3. Wolitski RJ, Fenton KA. Sexual Health, HIV, and sexually transmitted infectionas among gay, bisexual, and other men who have sex with men in the United States. AIDS Behav, 2011; 15: S9-S17.

4. Mayer KH. Sexually transmitted diseases in men who have sex with men. Clinical Infectious Diseases $2011 ; 53(\mathrm{~S} 3)$ :S79-83.

5. Gunn RA, O'Brien CJ, Lee MA, Gilchick RA. Gonorrhea Screening Among Men Who Have Sex With Men:Value of Multiple Anatomic Site Testing, San Diego, California, 1997-2003. Sexually Transmitted Diseases, October 2008; 35(10): 845-8.

6. Leone PA. Epidemiology, pathogenesis, and clinical manifestations of Neisseria gonorrhoeae infection. In: UpToDate, Basow, DS (Ed), UpToDate, Waltham, MA, 2013.

7. Bignell C, Unemo M. 2012 European guideline on the diagnosis and treatment of gonorrhoea in adults. Euro Surveill. 2012 Nov 22; 17(47).

8. Centers for Disease Control and Prevention. Update to CDC's Sexually Transmitted Diseases Treatment Guidelines, 2010: Oral Cephalosporins No Longer a Recommended Treatment for Gonococcal Infections. MMWR 2012;61:590-4.

9. Bébéar C, Barbeyrac B. Genital Chlamydia trachomatis infections. Clin Microbiol Infect 2009; 15: 4-10.

10. Zenilman JM. Genital Chlamydia trachomatis infections in men. In: UpToDate, Basow, DS (Ed), UpToDate, Waltham, MA, 2013.

11. Zenilman JM. Lymphogranuloma venereum. In: UpToDate, Basow, DS (Ed), UpToDate, Waltham, MA, 2013.

12. Lanjouw E, Ossewaarde JM, Stary A, Boag F, Meijden WI. 2010 European guideline for the management of Chlamydia trachomatis infections. Int J STD AIDS 2010 21: 729.

13. Centers for Disease Control and Prevention. Sexually Transmitted Diseases Treatment Guidelines, 2010. MMWR 2010;59:1-108.

14. Ng RAC, Samuel MC, Lo T, Bernstein KT, Aynalem G, Klausner JD et al. Sex, Drugs (Methamphetamines), and the Internet: Increasing Syphilis Among Men Who Have Sex With Men in California, 20042008. Am J Public Health. 2013;103:1450-6.

15. Mattei PL, Beachkofsky TM, Gilson RT, Wisco OJ. Syphilis: A Reemerging Infection. Am Fam Physician. 2012;86(5):433-40.

16. Wolff K, Johnson RA, Suurmond D, editors. 
Fitzpatrick's Color and synopsis of clinical dermatology. $5^{\text {th }}$ ed. New York: McGraw-Hill Medical; 2005. p. 911-24.

17. Abu-Raddad LJ, Margaret AS, Celum C, Wald A, Longini IM, Self SG et al. Genital Herpes Has Played a More Important Role than Any Other Sexually Transmitted Infection in Driving HIV Prevalence in Africa. PLoS ONE 3(5): e2230.

18. Albrecht MA. Epidemiology, clinical manifestations, and diagnosis of genital herpes simplex virus infection. In: UpToDate, Basow, DS (Ed), UpToDate, Waltham, MA, 2013.

19. Patel R, Alderson S, Geretti A, Nilsen A, Foley E, Lautenschlager $S$ et al. European guideline for the management of genital herpes, 2010. Int J STD AIDS. 2011 Jan;22(1):1-10.
20. Katz KA, Furnish TJ. Dermatology-Related Epidemiologic and Clinical Concerns of Men Who Have Sex With Men, Women Who Have Sex With Women, and Transgender Individuals. Arch Dermatol. 2005;141:1303-10.

21. Read TRH, Hocking JS, Vodstrcil LA, Tabrizi SN, McCullough MJ, Grulich AE et al. Oral Human Papillomavirus in Men Having Sex with Men: Risk-Factors and Sampling. PLoS ONE 7(11): e49324.

22. Lacey CJ, Woodhall SC, Wikstrom A, Ross J. 2012 European guideline for the management of anogenital warts. J Eur Acad Dermatol Venereol. 2012 Mar 12.

23. Makadon HJ. Primary care of gay men. In: UpToDate, Basow, DS (Ed), UpToDate, Waltham, MA, 2013. 